EPJ Web of Conferences 59, 03003 (2013)

DOI: $10.1051 /$ epjconf/20135903003

(C) Owned by the authors, published by EDP Sciences, 2013

\title{
Direct-drive shock-ignition for the Laser MégaJoule
}

\author{
B. Canaud ${ }^{1, a}$, V. Brandon ${ }^{1}$, S. Laffite ${ }^{1}$, M. Temporal ${ }^{2}$ and R. Ramis ${ }^{2}$ \\ ${ }^{1}$ CEA, DAM, DIF, 91297 Arpajon, France \\ ${ }^{2}$ ETSIA, Universidad Politécnica de Madrid, Spain
}

\begin{abstract}
We present a review of direct-drive shock ignition studies done as an alternative for the Laser MégaJoule (LMJ). One and two dimensional systematic analyses of HiPER-like shock-ignited target designs are performed for the fuel assembly irradiation uniformity using the whole LMJ configuration or a part of the facility, and for the uniformity of the ignitor spike. High-gain shock-ignition is shown to be possible with intensity of each quad less than $10^{15} \mathrm{~W} / \mathrm{cm}^{2}$ but low modes asymmetries displace the power required in the ignitor spike towards higher powers. Shock-ignition of Direct-Drive Double-Shell non-cryogenic targets is also addressed.
\end{abstract}

\section{INTRODUCTION}

Direct drive inertial fusion on the laser Megajoule (LMJ) [1-4] is complementary to the indirect drive [5] to study inertial confinement fusion (ICF) and to produce high thermonuclear energy and gain. In direct drive, laser beams overlap on the external side of a spherical target and the irradiation uniformity has to be addressed carefully [6-8] in order to improve target hydrodynamic stability [9, 10]. However, the LMJ beam layout for direct-drive self ignition requires zooming technique [4] and without it, the target should provide only marginal ignition. Recently [11], Shock Ignition was proposed as a nonisobaric ignition of the fuel which consists in separating the fuel assembly from the ignition produced by a convergent ignitor shock. This very promising solution was recently proposed to ignite the Fast Ignition capsule of HiPER $[12,13]$. We address here the possibility of using this approach for LMJ with HiPER-like targets by a 1D- and 2D-analysis [14, 15], considering the LMJ beam layout. Direct-drive shock-ignition of double shell targets [16] is also adressed.

\section{1D-ANALYSIS OF DIRECT-DRIVE SHOCK IGNITION FOR LMJ}

In order to obtain an adapted target design for LMJ we scale up [17] the HiPER target as shown in Fig.1. We then consider shock ignition of each target done by adding a timed laser spike. Calculations are done with the multi-dimensional Lagrangian radiation-hydrodynamics code FCI2 [18] in 1D.

The thermonuclear gain obtained for each target as a function of the spike power presents an ignitor power threshold above which the gain becomes maximum and then decreases as seen in Fig. 2a. Below it, the target is not igniting. This power threshold decreases when target approaches the isobaric selfignition threshold. This is due to the fact that targets closer to the isobaric ignition threshold (curve in black, on Fig. 2b) require lower peak ignitor power.

From this set of target designs we extract one (target $\mathrm{c}$ in Fig. 1) which matches the LMJ energy requirements for fuel assembly and shock ignition.

\footnotetext{
ae-mail: benoit.canaud@cea.fr
}

This is an Open Access article distributed under the terms of the Creative Commons Attribution License 2.0, which permits unrestricted use, distribution, and reproduction in any medium, provided the original work is properly cited. 

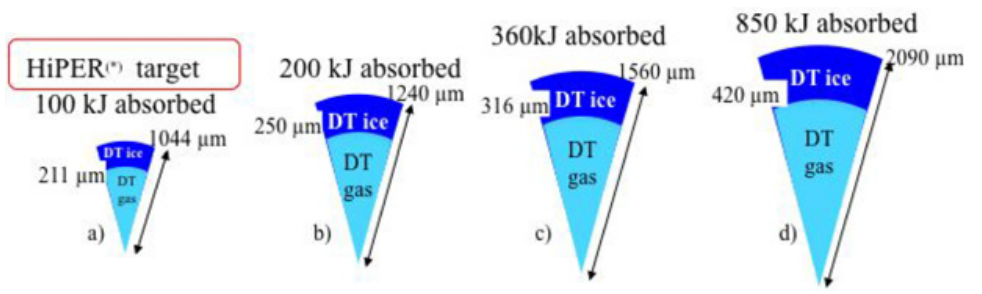

Figure 1. Different target designs produced by a scaling up the HiPER target.
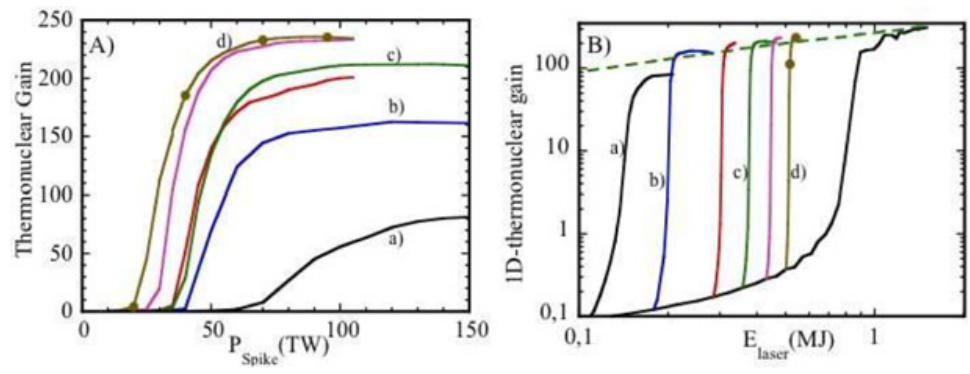

Figure 2. Shock-ignition and thermonuclear gain for each HiPER-like design versus the spike power (A) and the laser energy (B).

\section{2D ANALYSIS OF DIRECT-DRIVE SHOCK IGNITION FOR LMJ}

The LMJ will be a 240-beamlet frequency-trippled $(3 \omega-0.35 \mu \mathrm{m}) \mathrm{Nd}$-glass laser facility. Beams layout is fully described in Ref. [15] and are distributed on rings located at polar angles of $33.2^{\circ}, 49^{\circ}$ and $59.5^{\circ}$. We have showed [4] that DD-fuel assembly is possible with only the rings at $49^{\circ}$ and $59.5^{\circ}$, and $1 \mathrm{MJ}$. In addition, the ring at the polar angle of $33.2^{\circ}$ is unemployed in this configuration and could be used for shock ignition with a maximum laser power of $200 \mathrm{TW}$. The target ((c) in Fig. 1) implodes with a low in-flight adiabat $(\alpha \sim 0.8)$ and a peak implosion velocity of $290 \mathrm{~km} / \mathrm{s}$. At stagnation, the peak areal density is $\rho r \sim 19 \mathrm{~kg} / \mathrm{m}^{2}$ and peak density is $\rho \sim 680 \times 10^{3} \mathrm{~kg} / \mathrm{m}^{3}$. We first consider 1D-calculation of the fuel assembly with $1 \mathrm{D}$-centered laser beams and an additional laser beam located at a polar angle of $33.2^{\circ}$ to generate the ignitor spike. In our calculations, this last laser beam is modeled by 3D ray-tracing algorithm. The focal shape is a top-hat circular intensity profile with a radius $r_{0}$ varying from 0.8 to $1.2 \mathrm{~mm}$. The spike is a $300 \mathrm{ps}$ long flat laser pulse with $200 \mathrm{ps}$ rise and fall times. A variation of spike power $P_{\text {spike }}$ and pulse timing between driver and spike is performed to find the ignition window and to estimate the thermonuclear gain that is defined fully in Ref.[15]. As it can be seen, the smaller the focal spot, the better the laser-target coupling is, and the lower the ignitor spike power has to be. When the ignitor focal spot radius is larger, the ignitor shock is much more isotropic and less intensity is required in the spike to achieve ignition and gain. In addition, the increase of the radius leads to a quadratic reduction of the intensity needed to ignite the target. From Fig. 3b, it can be seen that the intensity in each quad is at a low level of a few $10^{14} \mathrm{~W} / \mathrm{cm}^{2}$ that is added to the fuel assembly intensity which is about $2 \times 10^{14} \mathrm{~W} / \mathrm{cm}^{2}$. Nevertheless, the resulting intensity stays at low level, well below $10^{15} \mathrm{~W} / \mathrm{cm}^{2}$. These results show that choosing a target design not so far from the self-ignition threshold allows to reduce the intensity of the ignitor spike at level of the order of the two-plasmon decay threshold. We then consider the full 2D problem of fuel assembly and shock ignition by using two rings located at polar angles of $49^{\circ}$ and $59.5^{\circ}$ for fuel assembly and $33.2^{\circ}$ for shock ignition. Calculations used a top-hat intensity profile, a focal spot radius equal to $1.4 \mathrm{~mm}$, and an equal ring-to-ring power balance. The laser pulse is reshaped in comparison to the 1D-pulse in order to take into account the $3 \mathrm{D}$ aspect of the laser 

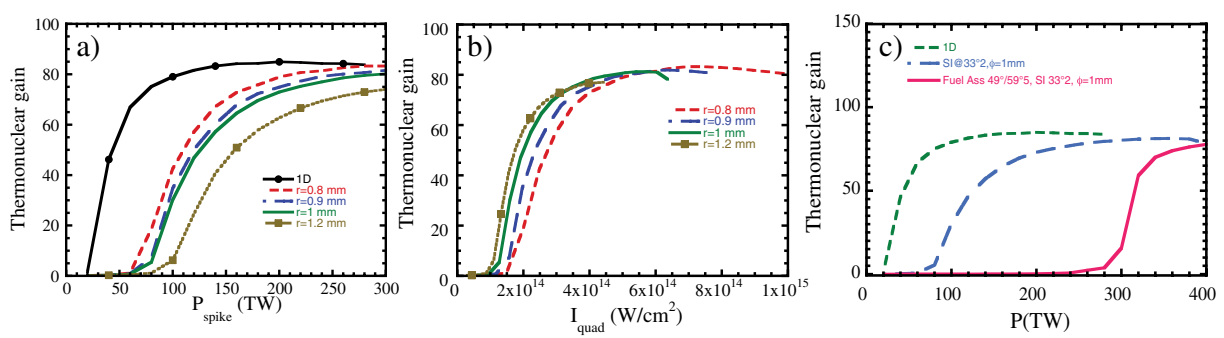

Figure 3. 1D-Thermonuclear gain versus the spike power (a) and spike intensity (b) of each quad for the ring at $33.2^{\circ}$ for different focal spot radii for the full-1D calculation (plain line and circles). Thermonuclear gain versus the spike power (c) for previous cases and for full 2D calculations.
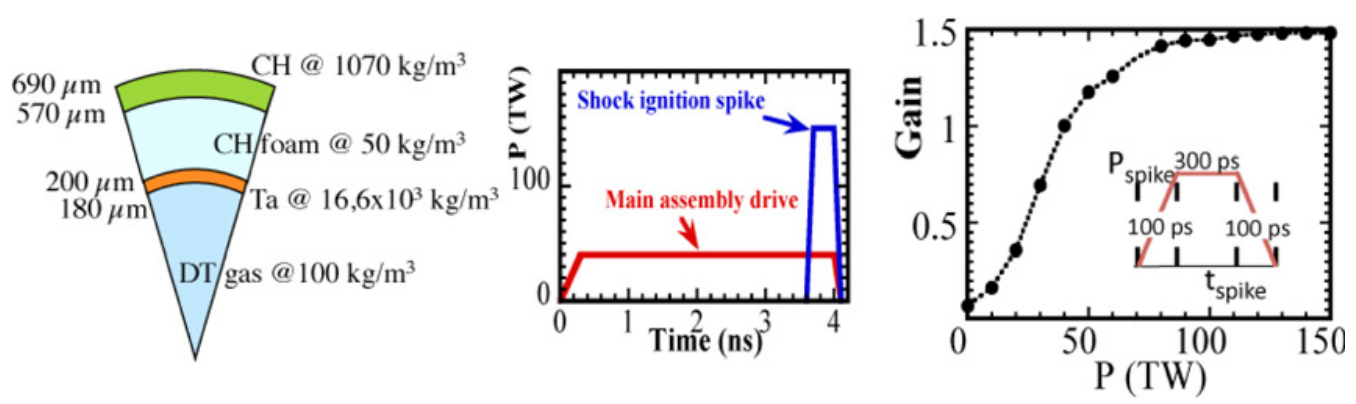

Figure 4. Direct-drive double-shell design (left).Thermonuclear gain (right) versus ignitor spike power.

beams (especially the energy losses by the side of the imploding target). The irradiation uniformity is not fine but sufficient to ensure a relatively good isotropy of the implosion. However, a reduction of the peak density and areal density is observed due to the anisotropy of the stagnating shell. Indeed, the areal density changes from $18.5 \mathrm{~kg} / \mathrm{m}^{3}$ to $16 \mathrm{~kg} / \mathrm{m}^{2}$ with a rms deviation of $37 \%$. The implosion velocity and the in-flight adiabat are kept similar to the $1 \mathrm{D}$ ones. Shock ignition is done by the $33.2^{\circ}-$ polar angle beams with a top-hat intensity profile radius of $1 \mathrm{~mm}$. Fig. 3c shows a comparison of shock ignition for the full-1D, for 1D-fuel assembly and 2D shock ignition, and for the full 2D calculations. It can be seen that high thermonuclear gain is achieved for higher spike power in full 2D-calculations. This power increase is mainly due to strong low mode asymmetries observed at stagnation due to the full assembly done in a quasi-direct drive beam geometry. These asymmetries displace the self-ignition threshold towards higher energies as observed previously [4]. As the self-ignition threshold is displaced towards higher kinetic energy values, the spike power needed for shock ignition is much higher.

\section{SHOCK IGNITION OF DIRECT-DRIVE DOUBLE-SHELL TARGET}

Shock ignition is investigated for non-cryogenic direct-drive double-shell targets [16]. This concept consists in accelerating an external shell (see Fig. 4) to high velocity (few hundreds $\mathrm{km} / \mathrm{s}$ ) that goes to impact a high-Z inner shell enclosing the DT gas. The fuel assembly is obtained by direct laser light absorbed in the external shell. The ignition is produced by a spherically convergent shock launched by a laser spike added at the end of the main laser drive. A moderate thermonuclear gain (1-10) is obtained for a well-synchronized, high-pressure ignitor shock. The latter is produced by a $300 \mathrm{ps}$ few hundred TW laser spike added at the end of the drive pulse. We have shown also that keeping the main drive constant implies a modification of the peak implosion velocity and leads to a lower ignition spike power threshold than the one needed when the implosion velocity is kept constant by reducing the main drive. 
EPJ Web of Conferences

\section{CONCLUSIONS}

Direct-drive shock ignition is proposed for LMJ for cryogenic HiPER-like target and double shell noncryogenic target. High gain can be achieved with shock ignition for cryogenic targets. Shock ignition is achieved for different targets of the fast ignition family which are driven by an absorbed energy between $100 \mathrm{~kJ}$ and $850 \mathrm{~kJ}$ and are delivering thermonuclear energies between 10 and $130 \mathrm{MJ}$. Moderate gains are achieved for double-shell targets. 2D calculations show that high-gain shock-ignition is possible with intensity of each quad less than $10^{15} \mathrm{~W} / \mathrm{cm}^{2}$ but low modes asymmetries displace the ignitor spike power towards higher powers.

\section{References}

[1] B. Canaud, X. Fortin, F. Garaude, C. Meyer, F. Philippe, Las. Part. Beams 22, 109 (2004)

[2] B. Canaud and F. Garaude, Nucl. Fusion 45, L43 (2005)

[3] D. Riz, F. Garaude, M. Houry, and B. Canaud, Nucl. Fusion 46, 864 (2006)

[4] B. Canaud, F Garaude, C Clique, N Lecler, A Masson, R Quach, J Van der Vliet, Nucl. Fusion 47, 1652 (2007)

[5] J. Giorla, J. Bastian, C. Bayer, B. Canaud, M. Casanova, F. Chaland, C. Cherfils, C. Clique, E. Dattolo, P. Fremerye, D. Galmiche, F. Garaude, P. Gauthier, S. Laffite, N. Lecler, S. Liberatore, P. Loiseau, G. Malinie, L. Masse, A. Masson, M. C. Monteil, F. Poggi, R. Quach, F. Renaud, Y. Saillard, P. Seytor, M. Vandenboomgaerde, J. Van der Vliet and F. Wagon, Plasm. Phys. Control. Fusion 48, B75 (2006)

[6] B. Canaud, X. Fortin, N. Dague and J.L. Bocher, Phys. Plasmas 9, 4252 (2002)

[7] M. Temporal, and B. Canaud, Eur. Phys. J. D 55, 139 (2009)

[8] M. Temporal, R. Ramis, and B. Canaud, J. Phys.: Conf. Series 244, 022008 (2010)

[9] M. Temporal, S. Jaouen, L. Masse, and B. Canaud, Phys. Plasmas 13, 122701 (2006)

[10] J. Sanz, J. Garnier, C. Cherfils-Clerouin, B. Canaud, L. Masse, and M. Temporal, Phys. Plasmas 12, 112702 (2005)

[11] R. Betti, C. Zhou, K. Anderson, L. Perkins, W. Theobald, and A. Solodov, Phys. Rev. Lett. 98, 155001(2007)

[12] S. Atzeni, A. Schiavi, and C. Bellei, Phys. Plasmas 14, 2702 (2007)

[13] X. Ribeyre, G. Schurtz, M. Lafon, S. Galera, and S. Weber, Plasm. Phys. Control. Fusion 51, 015013 (2009)

[14] B. Canaud and M. Temporal, New J. Phys. 12, 3037 (2010)

[15] B. Canaud, S. Laffite, V. Brandon, and M. Temporal, Las. Part. Beams 30, 183 (2012)

[16] B. Canaud, S. Laffite, and M. Temporal, Nucl. Fusion 51, 062001 (2011)

[17] E. Falize, C. Michaut, and S. Bouquet, Astrophys. J. 730, 96 (2011)

[18] E. Buresi, J. Coutant, and R. Dautray, Las. Part. Beams 4, 531(1986) 